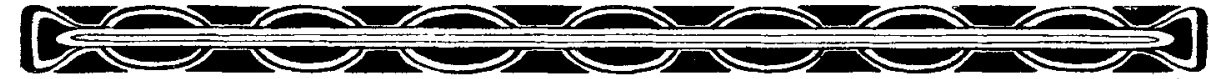

\title{
Liste der an die Redaktion eingesandten Bücher, Separata und Periodica, soweit sie im Jahre 1909') erschienen sind.
}

\section{A. Bücher. $\left.{ }^{2}\right)$}

Brauer, F., Die Süßwasserfauua Deutschlands. Eine Exkursionsfauna. (Jena 1909, Gust. Fischer.)

Heft 1. Matschie, Mummalia, 14 S., 4 $\Lambda$ bb.

Reichenow, Aves, $49 \mathrm{~S}$., $40 \mathrm{Abb}$.

T'ornier, Reptilia, Amphibia, 26 s., $130 \mathrm{Abb}$.

Pappenheim, Pisces, 112 S., 76 Abb.

"3/4. Reitter, Coleoptera, 235 S., 101 Aub.

" 5/6. Ulmer, Trichoptera, 326 S., 467 Abb.

"7. Heymons, Collembola, Neuroptera, Hymenoptera, 36 S., 55 Abb.

Kühlgatz, Rynchota, 74 S., 56 Abb.

" 8. Klapalek, Ephemerida, Plecoptera, 95 S., 177 Abb.

Grünberg, Lepidoptera, 64 S., 83 Abb.

"9. Ris, Odonata. $67 \mathrm{~S} ., 76 \mathrm{Abb}$.

"10. Keilhack, Phyllopoda.

"11. van Douwe, Copepoda, freilebende, 69 S., 310 Abb.

Neresheimer, Copepoda, parasitische, 15 S., 35 Abb.

Vávra, Ostrucoda, 35 S., 139 Abb

K eilhack, Malacostraca, 13 S., 21 Abb.

- 12. Dahl, Araneae, 12 S., $6 \mathrm{Abb}$

Koenike, Acarina, 172 S., 271 Abb.

Brauer, Tardigrada, 2 S., 3 Abb.

"13. Michaels en, Oligochaeta, 66 S., $112 \mathrm{Abb}$.

Johansson, Hirudinea, 15 S., 32 Abb.

"15. Jägerskiöld, Freilebende Süßwassernematoden, 46 S., 65 Abb.

v. Linstow, Parasitische Nematoden, 37 S., 80 Abb.

Hartmeyer, Mermithidae, Gurdiidae, 5 S., 10 Abb.

17. Lühe, Parasitische Plattwürmer, 1. Trematodes, 217 S., 188 Abb.

" 18. Lüh e, Parasitische Plittwürmer, 2. Cestodes, $153 \mathrm{~S}, 174 \mathrm{Abb}$.

$" 19$. Thiele, Mollusch, 46 S., $112 \mathrm{Abb}$.

Hartmeyer, Nemertini, Bryozoa. $12 \mathrm{~S} ., 19 \mathrm{Abb}$.

v. Graff, Turbellaria, 84 S., $120 \mathrm{Abb}$.

Böhmig, Tricladida, 34 S., 41 Abb.

Weltner. Spongillidae, $14 \$, 40 \mathrm{Abb}$.

Brauer, Hydrozoa 4 S.. 14 Abb.

Fechner und Paeske, Fischkalender: Bd. I, Fische unserer Flüsse und Seen. 32 S. mit vielen farbigen Abbild. Leipzig 1909 Verlag F. W. Grunow.

Fennemann, N. M., On the lakes of Southeastern Wisconsin. Wisconsin geological and nat. history Survey. Madison, 1902. 178 S., 36 Taf., 38 Fig. im Text.

Hentschel, E., Das Leben des Süßwassers. Reinhardts Verlag, München 1909, 336 S., 228 Abb. im Text, 16 Vollbilder, 1 farb. Titelbild. [Referat in dieser Revue, S. 483.]

1) Eine große Zahl von Einsendungen beziehen sich auf das Jahr 1908, diese sind in den "Jahresübersichten für 1908", aber nicht nochmals in dieser Liste aufgeführt. Andere stammen aus 1907 oder früheren Jahren; auch diese muBten aus Raummangel hier fortgela:sen werden, doch kommen wir auf die wichtigsten dieser früheren Arbeiten, so auf eine Serie hydrologischer Schriften Finnlands. im Referatteil des III. Bandes noch zurüek.

2) Nur von diesen sind auch solche aufgeführt, welche vor 1909 erschienen sind, aber wegen ihrer Wichtigkeit noch referiert werden sollen.

Revue d. ges. Hydrobiol. u. Hydrogr. Bd. II. H. 6. 
Hjort, J., Report on Norwegian Fishery and Marine Investigations. Vol. II, Part I and II, Bergen 1909. Enthält: Oversigt over Norsk Fiskeri- \& havforskning 1900-1908, (204 S.). Ferner:

Helland-Hansen and Nansen, The Norwegian Sea, its Physical Oceanography based upon the the Norw. Researches 1900-1904, (360 S., 112 Textfig., 3 Tabellen, 28 Tafeln).

Collet, Fiske, indsamlede under "Michael Sars"s togter i Nordhavet 1900-1902, (151 S., 2 Taf.).

Sandström and Helland-Hansen, Über die Berechnung von Meeresströmungen, (43) S.).

Gran, Das Plankton des Norwegischen Nordmeeres, (122 S., 1 Taf.).

Dahl, The scales of the herring as a means of determining age, growth and migration (36 S., 3 Taf.).

Jaeger, G., Das Leben im Wasser und das Aquarium. Kosmos Stuttgart, 367 S., 9 Taf., 151 Fig. im Text.

Johnstone, J., Conditions of Life in the Sea. Cambridge Biolog. Series, 332 S., 31 Fig. im Text, 1 farbige Karte. 1908.

Katzer, Karst und Karsthydrographie. Serajewo 1909.

Knauthe, K., Das Süßwasser. Chemische, biologische und bakteriologische Untersuchungsmethoden unter besonderer Berücksichtigung der Biologie und der fischereiwirtschaftlichen Praxis. Verlag Neumann in Neudamm, 1907. 663 S., 194 Fig. im Text.

v. Knebel, W., Höhlenkunde mit Berücksichtigung der Karstphänomene. Die Wissenschaft, Sammlung naturwiss. u. math. Monograph. Verlag Vieweg \& Sohn in Braunschweig 1906, Heft 15, $222 \mathrm{~S}, 42$ Abbild. im Text u. auf 4 Taf.

Philippe Duc d'orléans, Croisière Océanographique accomplie à bord de la Belgica dans la mer du Grönland 1903̆. Bruxelles 1907. 568 S., 79 Tafeln, Großquart. Enthält 11. a.:

de Gerlache, Relation du voyage. Jounal de Bord (61 S.).

Böggild, Sédiments sous-marins.

Ostenfeld, Plantes.

Helland-Hansen and Koefoed, Hydrographie (72 S.).

Koefoed, Plankton (106 S.).

Jörgensen, Radiolaires.

Hartlaub, Méduses.

Ko efoed, Poissons.

Grieg, Invertíbrés du fond $(70 \mathrm{~s}$.$) .$

Marsh, C. Dwight, The Plankton of Lake Winnebago and Green Lake. Wisconsin Geolog, and Nat. Hist. Survey. Madison 1903, 94 S., 22 Taf.

Steuer, A., Planktonkunde. Verlag B. G. Teubner. 723 S., 360 'Textabbild., 1 Taf.

Verworn, M., Allgemeine Physiologie. 5. Aufl. 1909, 742 S., 319 Abbild. Jena, G. Fischer. [Referat in dieser Revue, Bd. II, S. 784.]

Hyadrobiologie in Schulbüchern:

Heering, W., Leitfaden für den biologischen Unterricht. Weidmannsche Buchhandlung Berlin 1908, 319 S., 206 Abbild.

Schmid, B., Biologisches Praktikum für höhere Schulen. Verlag B. G. Teubner. Leipzig 1909, 71 S., 9 'Taf., 75 Fig. im Text.

Schurig, W., Biologische Experimente. Quelle u. Meyer, Leipzig 1909. 180 S., 87 Abb.

Voigt, M., Die Praxis des naturkundlichen Unterrichts. Ein Handbucl für Lehrer aller Schulgattungen und für Sammler. Verlag Dieterich, Leipzig 1909, 282 S., 92 Figg. im Text.

\section{B. Eingesandte Periodica.}

\section{Berichte der Stationen, Museen, Institute etc.}

Batavia, Mededeelingen van het Visscherij-Station te, Nr. III:

van Kampen, Aanteekeningen omtrent de Visscherij van Sumatra en Riouw, $29 \mathrm{~s}$.

Bergen's Museums Aarbog 1909. Enthält u. a.:

Nordgaard, Studier over naturfoholdene i vestlandske fjorder, II., 20 S. [Hydrographie und Plankton.]

Landmark, Reliefstudier fra Søndfjord, 44 S., 3 Karten, 37 Textfig. 
Berkeley, University of California Publication 1909: Physiology Vol. III, Botany Vol. III, Zoology Vol. VI. [Vergl. Liste C.]

Berlin, Arbeiten aus dem Kais. Gesundheitsamt zu -. Bd. XXXII-XXXIII. [Siehe Liste C.]

Besse, Annales de la Station Limnologique de - Fase. I. et II., Janvier-Avril 1909. Enthält außer vielen historischen, geographischen, floristischen, entomologischen Aufsätzen und Notizen eine Bibliographie für die Naturgeschichte der Auvergne. Ferner:

Bruyant, Note sur la présence de Planaria alpina en Auvergne.

Calcutta, Records of the Indian Museum, Vol. II-III, 1909. Enthālt u. a.: Annandale, Notes on Freshwater Sponges, X, XI.

Annandale, A pelagic See-Anemone without tentacles.

Annandale, Description of a Barnacle of the genus Scapellum.

Annandale, Prelim. note a new genus of Phylactolaematous Polyzoa.

Stephenson, Preston, Germain, Stewart, Report on a collection of aquatic animals made in Tibet 1907 . II.

Alcock, Diagnoses of new species and varieties of Freshwater Crabs.

Milano, Atti della Società Italiana die Scienze Naturali e del Museocivico di Storia Naturale in -, Vol. XLVII, 1909. Enthält u. a.:

Supino, I cosi detti pesci antimalarici. $4 \mathrm{~S}$.

Barbieri, Neuromeri e somiti meta-otici in embrioni di Salmonidi.

Monaco, Bulletin de l'Institut Océanographique. No. 131-153, Janvier-September 1909. [Vergl. Liste C.]

San Diego, Contributions from the Laboratory of the Marine Biological Association of -. XXV-XXVIII. (University of California Publications in Zoology.) [Vergl. Liste C.]

\section{Hydrobiologie, Fischerei, Hydrographie:}

Annales de Biologie Lacustre Tome III, fasc. 3 (Mars 1909). [Vergl. Liste C.]

Archiv fūr Hydrobiologie und Planktonkunde. Bd. IV, H. 3 (mit 9 Textfig.), H. 4 (mit 3 Taf. u. 4 Textfig.); Bd. V, H. 1 (mit 2 Taf. u. 32 Textfig.) [Enthält außer den in Liste $C$ aufgeführten Originalaufsätzen verschiedene Autorreferate, Referate und Notizen.]

Allgemeine Fischereizeitung, München, Jahrgang XXXIV. [Enthält zahlreiche Origina!aufsätze und -notizen, Bekanntmachungen, Vereinsnachrichten, Marktberichte und Literaturbesprechungen. Vergl. auch Liste C.]

Bulletin Suisse de Pêche et Pisciculture. Neuchàtel X. Année, 1909. (12 Hefte) [Enthält: Originalartikel und -notizen, Gesellschaftsberichte, Bekanntmachungen, Bibliographie, Praktische Winke, Rezepte, Feuilleton].

Fischereizeitung, Schweizerische, Pfäffikon. 17. Jahrgang 1909. (12 Hefte). [Enthält: Originalaufsätze, Fischerei-Neuheiten, Vereinsberichte, Vermischtes, Fragekasten, Literatur].

Fischerbote, Der. Hamburg. Jahrgang I.: 1909 (Monatsschrift). [Enthält: Originalaufsätze, Fischerei-Nachrichten, Fischmarktberichte, Vereinsberichte, „vom Leben unserer Fischer", Fischverwertung, Bekanntmachungen].

osterreichisehe Fisehereizeitung. Wien. V. Jahrgang 1909. (24 Hefte). [Enthält: Originalaufsätze, Fischerei-Nachrichten (See- und Binnenfischerei). "Angler-Ecke," „aus fremden Zeitschriften“, Vereinsnachrichten, Vermischtes, Marktberichte. Vergl. auch Liste C].

Zeitschrift für Fischerei und deren Hilfswissenschaften, Berlin. XV. Band, 19081909. [Enthält nur größere wissenschaftliche Originalaufsätze].

Wasser und Abwasser, Berlin. Bd. I. (24 Hefte). [Enthält Originalberichte, aber vor allem zahlreiche Referate].

Publications de Cireonstanee du Conseil Permanent. International pour l'Exploration de la Mer. Copenhagen, 1909. [Siehe Liste C].

3. Allgemeine Biologie, Botanik, Zoologie:

Acta Societatis pro Fauna et Flora Fennica. Helsingfors 1909: Bd. XXIV. [Enthält: "Bibliotheca Zoologica Fenniae" bis 1901. 361 S.!] Bd. XXXII. [Vergleiche Liste C.] 
Archiv Pür Rassen-uad Gesellschaftsbiologie. Leipzig. VI. Jahrgang (6 starke Hefte.) Enthalt $u$. a.:

Hase, Utber die deutschen Süßwasserpolypen Hydra tusca. H. grisea und H. viridis. (33 S., 10 Fig.); [außerdem zahlreiche Abhandlungen, Notizen und Referate].

Bulletin Biologique. Dorpat. 3. Jahrgang 1909 (monatlich 2 Hefte). [Enthält in deutscher und russischer Sprache wissenschaftliche Mitteilungen, Sitzungsberichte, Referate und "Auskunftsteil “, in letzterem viele Notizen über Stationen, Expeditionen u. dgl.]

Bulletin de la Société Loologique de France. Paris. Tome XXXIV (Nr. 1-9). Futhält u. a.:

de Beauchamp, Infusoires du poumon des Holothuries is Banyuls.

Gulia, Cephaloptera giorna [Plagiostomes] dans les eanx maltaises.

Gulia, addition à la faune ichthyologique maltaise.

Chatton, Ophioseides, Copépode parasite de Microcosmus.

Chatton, Une Ascidie fixée dans la peau d'Holothuria.

de Beauch amp, Philodina intermedia, et remarques sur l'origines des Microdinidés (Rotafères).

de Beauchamp, Plagiostoma Lemani et Polycelis felina aux environs de Paris.

Danois, Gobius pictus en France.

Caziot, Mollusques terrestres et fluviatiles des Alpes maritimes.

Pellegrin, Poissons nouveaux de l'Afrique orientale anglaise.

Borcéa, (irculation embryomnaire chez les Téléostéens.

Chatton \&Brément, Enteropsis roscoffensis, Copépode parasite de Styelopsis (Ascidie).

Meddelanden af Soc. pro Fauna et Flora Fennica Helsingtors. XXV. Bd. 1908--1909. Enthält sehr zahlreiche kürzere Mitteilungen, u. a.:

Särvi, Beobachtungen' über die Größe und das Alter der Aale in Binnengewässer'n Finnlands.

Särvi, Über das Vorkommen des Aales im Wassersystem des Kymiflusses.

Särvi, Hermaphroditismus bei Lota vulgaris.

Häyrén, Algologische Notizen aus der Gegend von Björneborg.

Luther, Hermafrodiska exemplar af Lota vulgaris.

Hirs ch mann, Beitrag zurKenntnis der Ostrakodenfauna des Finnischen Meerbusens.

Memoires de la Société Zoologique de France. Paris 1909. Tome XXI-XXII. Enthält u. a.:

Blaizot, Recherehes sur l'évolution de l'utérus d'Acanthias vulgaris (47 $\mathrm{s.}$. 3. Taf.)

Caziot, Indication des ouvrages qui ont paru sur la faune des Mollusques terrestres et fluviatiles des départements trançais (30 S.).

Caziot. Dito: Algérie, Tunisie, Maroc (11 S.).

Vlès, Monographie sommaire de la Mye, (Mya arenaria L.) (53 s., 35 'T'extfig., 2 Tat.).

de Man, Note sur quelques espéces du genre Alphous Fabr, appartenant an groupe brevirostris de M. (19 S., 2 Taf.).

Nyt Magazin for Naturvidenskaberne. Kristiania 1909, Bind XLVII, H. 1-3. Enthallt u. a.:

Broch, Hydroiduntersuchungen II. (Bonneviella und Lictorella; $10 \mathrm{~S}$. mit 6 Textfiguren.)

Wille, Algologische Notizen XV. (Wittrockiella nov. gen.; 16 S. mit 4 Taf.)

Wollebaek, Giffektiv hermaphroditisme kos en decapod Crustace, Calearis Macandeae. (18 S., 3 Taf.)

Wahlstedt, Förteckning öfver Norska Characeer (4 S.).

Zeitschrift für Botanik. Jena, I. Jahrgang (12 Helte). [Enthält Originalaufsätze und -notizen, Referate und Listen der, Neuen Literatur".]

Gaea, Natur und Lehen. Stuttgart. XLV. Jahrgang 1909 (12 Hefte). [Orientierende Aufsãtze, vorwiegend geo- und hydrographischen Inlalts, Vermischte Nachrichten, Literaturbesprechungen].

Mikrokosmos. Stuttgart. III. Jahrgang 1909/10 (12 Hefte). [Populäre Aufsätze „mikrologischen" Inhalts, prakt. Winke, Referatc, Briefliasten].

Dio Klein welt. Bamberg. I. Jahrgang 1909/10 (12 Hofte). [Inhalt ähnlich dem Vorigen]. 
C. Separata: I. Ozeanographie und Hydrographie; Allgemeines.

Chevallier et Sodry, La source de la Bise dans l'Etang de Thau. Bull. Inst. Océan. Monaco Nr. 133, 17 S., 1 Taf.

Ekman, E. W., Die Zusammendrückbarkeit des Meerwassers nebst einigen Werten für Wasser und Quecksilber. Publications de Circonstance, No. 43, 47 S., 8 Fig.

Fox, Chas. J. J., On the Coefficient of Absorptions of the Atmospheric Gases in Destilled Water and Sea Water. II. Carbonic Acid. Publications de Circonstance, No. 44, 31 S., 5 Fig.

Gehrke, J., Úber Farbe und Durchsichtigkeit des Ostseewassers. Mit einer allgemeinen Theorie des Zusammenhanges zwisehen Farbe und Durchsichtigkeit in natürliehen Gewässern. Publications de Circonstance, No. 45, $20 \mathrm{~S}$.

Götzinger, G., Die Lunzer Seen. Mitteil. d. K. K. Geogr. Gesellsch. Wien 1909. $5 \mathrm{~S}$.

Halbfass, W., Die Ergebnisse der Seenforschung in Schottland. "Globus", Bd. XCV, S. $349-353$.

Legendre, P., Recherches physico-chimiques sur l'eau de la côte, à Concarneau. Bull. Inst. Océan. Monaco, Nr. 144, $29 \mathrm{~S}$.

Luther, Alex., Ưber eine Littorina-Ablagerung bei Tvärminne, nebst einigen Bemerkungen über die kalkauflösenden Eigenschaften der jetzigen Ostsee und des Littorinameeres. Acta soc. pro fauna et flora Fennica, Bd, XXXII, Nr. 4, 22 S., 2 Fig. im Text.

Marini, L., Quelques considérations sur le programme pour l'exploration internationale de l'Océan Atlantique et de la Méditerranée. Bull. Inst. Océan. Monaco, Nr. 143, $4 \mathrm{~S}$.

Nathansohn, A., Sur les relations qui existent entre les changements du plankton végétal et les phénomènes hydrographiques, d'après les recherches faites à bord de l'Eider, au large de Monaco, en 1907-1908. Avec 10 Planches. Bull. Inst. Océan. Monaco, Nr. 140. $90 \mathrm{~s}$. [Deutseh in dieser Revue, S. 580.]

Pettersson, 0., Gezeitenähnliche Bewegungen des Tiefenwassers. Publications de Circonstance, No. 47, 21 S., 1 Taf.

Thienemann, A., Eine einfache Form der Meyerschen Schöpfflasche. Arch. f. Hydrobiol. u. Planktonk., Bd. V, 4 S., 1 Fig. im 'Text.

Vinciguerra, M. le, Sur l'opportunité d'une exploration océanographique de la Méditerranée dans l'intérêt des pêches maritimes. Bull. Inst. Océan. Monaco, Nr, 139, $10 \mathrm{~s}$.

Wyss, M. O., Die Herbstiris der Seen. Revue Suisse de Zool., T. XVII, S. 441-447, 1 Taf.

Kofoid, Ch. A., Internationale Revue der ges. Hydrobiologie u. Hydrographie. Referat. Science. Vol. XXX, 1909. S. $684-687$.

Wolf, M., Dito. Referat. Biol. Centralbl., Bd. XXIX, S. 544-554.

\section{Allgemeine Biologie des Meeres.}

Albert I ex Prince de Monaco, La pêche dans les abîmes. Bull. Inst. Océan. Monaco. Nr, $137,15 \mathrm{~s}$.

Herdman, W. A., Our Food from the Waters. British Association, 31. Aug. Winnepeg 1909, 9. S.

Sernow, S. A., Kurzer historischer Uberblick über die Tätigkeit der Biologischen Station der Kaiserl. Akademie d. Wissenschaften in Sewastopol. Bulletin Biologique 1909. S. $280-285$.

Sernow, (Zernov), S. A., Compte-rendu de l'expédition pour l'exploration faunistique de la partie N.-W. de la mer Noire. Separat (russisch), 13 S., 1 Karte.

Sernow (Zernov), S. A., Sur la facies de la Phyllophora (Algae-Rhodoph.) dans la partie N.-W. de la mer Noire. Annuaire du Musée Zool. St. Pétersbourg, S. 181-191, $2 \mathrm{Abb}$. [Russisch; deutsches Resumé erscheint in Bd. III dieser Revue.]

Steuer, A., Ein Vorschlag zur Hebung der österreichischen Korallenfischerei. Osterr. Fisch.-Zeitg., Jahrg. VII, 12 S., 4 Fig.

Vauhöffen, E., Tiere und Pflanzen von St. Paul und Neu-Amsterdam. Deutsche Sũdpolarexpedition 1901-1908, Bd. II, Geogr. u. Geol., S. 401-410.

Ziegler, H. E., An den Buhnen von Norderney. Westermanns Mlonatshefte August 1909, 7 S., 10 z. T. farb. Abb. 


\section{Allgemeine Biologie des $\mathrm{SüB}$ wassers.}

Brehm, V., Uber die Mikrofauna chinesischer und südasiatischer Süßwasserbecken. Arch. f. Hydrob. u. Planktonk., Bd. IV, 17 S., 7 Fig. im Text.

Keilhack Lndwig, Beiträge zur Kenntnis der Süßwasserfauna der Dauphiné-Alpen. Arch. f. Hydrob. u. Planktonk., Bd. IV, 14 S., 1 Taf.

Kolkwitz, R., Ober die Planktonproduktion der Gewässer, erläutert an Oscillatoria Agardhii Gom. Landwirtschaftl. Jahrb. Erg., Bd. V, S. 449-472, 1 Tafel, 2 Textabbild.

Ianghans, V. H., Planktonprobleme. Lotos, Bd. LVII, 6., $12 \mathrm{~S}$.

Lanterborn, R., Bericht über die Ergebnisse der 6. biologischen Untersuchung des Oberrheins auf der Strecke Basel-Mainz (vom 15. bis 30. Nov. 1907.) Arb. a. d. Kais. Gesundheitsamt, Bd. XXXII, S. $25-58$.

Marsson, M., Bericht über die Ergebnisse der vom 29. Nov, bis zum 7. Dez. 1907 ausgeführten 6. biologischen Untersuchung des Rheins auf der Strecke Mainz bis Coblenz. Ibidem, S. $58-88$.

Stazione di Biologia e di Idrobiologia Applicata Acquario-Milano. 15 S., 7 Abb.

Thienemann, A., Orphuephila Testacea Macq. Ein Beitrag zur Kenntnis der Fauna hygropetrica. Ann. Biol, lacustre, T. IV, S. 53-86, 3 Fig., 2 Taf.

Wesenberg-Lund, C., Grundtraekkene i Ferskvands planktonets Biologi og Geografi. Ymer, Stockholm, 44 S., 19 Fig. im Text. [Erscheint deutsch in Bd. III dieser Revue.]

Zacharias, 0., Das Süßwasser-Plankton als Gegenstand der naturkundlichen Unterweisung in der Schule. 0 . Kaoens Buchdruckerei, Plön i. Holst., 8. S.

\section{Botanik des Meeres und Süßwassers.}

Forti, A., Studi per una Monografia del genere Pyxilla (Diatomee) e dei generi affini Nuova Notarisia, Serie XX, 20 S., 3 'Taf.

Crardner, N. L., New Cllorophyceae from California. University Calif. Publ. Botany. Vol. III, S. $371-376,1$ Taf.

Kofoid, Ch. A., Reports on the Scientific Results of the Expedition to the Eastem Tropical Pacific, in charge of Alexander Agassiz, by the U. S. fish Commission Steamer "Albatross", XX. Mutations in Ceratium. Bull. of the Mus. of Comp. Zool. Harvard College vol. LI, S. 212-257, 4 Taf., 5 Fig., 1 Tabelle.

Kofoid, Ch. A., On Peridinium steini, Jörgensen, with a note on the nomenclature of the skeleton of the Peridinidae. Arch. f. Protistenk, Bd. XVI, S. 25-47, 1 T.

Kofoid, Ch. A, The morphology of the skeleton of Podolampas. Ibidem Bd. XVI, S. 4861,1 Taf.

Nichols, M. B., Contributions of the Knowledge of the California species of Crustaceous Coralline. II. University Calif. Publ, Botany, Vol. III, S. 349-370, 4 'Taf.

Reichenow, Ea., Tntersuchungen an Haematoccocus pluvialis und eingen anderen Flagellaten. Sitz.-Ber. Gesellsch. naturf. Freunde Berlin, Jahrg. 1909, \&. 85-91, 2 Fig. im Text.

Reichenow, Ed.. Untersuchungen an Haematococcus pluvialis nebst Bemerkungen über andere Flagellaten. Arch. a. d. Kaiserl. Gesundheitsamt Berlin, Bd. XXXIII, 45 S., 2 Taf.

Richter, 0., Zur Physiologie der Diatomeen. II. Mitt. Die Biologie der Nitzschia putrida Benecke. Denkschr. K. Acad. Wissensch. Wien Math.-nat. Klasse, Bd. LXXXIV, 116 S., 4 Taf., 6 Fig. im Text, 2 Haupt- u. 7 Texttabellen.

Sehiller, J., Ein neuer Fall von Mikrosporenbildung bei Chaetoceras Lorenzianum Grun. Ber. der Deutsch. Bot. Gesellsch, Bd. XXVII, S. 351-361, 1 'Taf.

Protozoen:

$$
\text { V. Einzelne Tiergruppen des Meeres und SüBwassers. }
$$

Borgert, A., Über Erscheinungen fottiger Degenerationen bei tripyleen Radiolarien. Arch. f. Protistenk., Bd, XVI, S. 1-24, 1 Taf., 4 T'extfig.

Hartmann, M. u. Hammer, E., Untersuchungen über die Fortpflanzung von Radiolarien. Sitzungsher. d. Ges. f. Naturf. u. Fr., Nr, 4, S. 228-248, 1 Taf., 4 Textfig.

Martin, B. A., Some Observations on Acinetaria. Quart. Journ. Micr. Sc., Vol. LIII, part. 2, S. 351-389, 2 Taf., 12 Fig. im Text.

Nägler, K., Entwicklungsgeschichtliche Studien über Amöben. Arch. f. Protistenkunde, Bd. XV, S. 1-53, 6 Taf. 
Schmidt, I. W., Beobachtungen über den Bau und die Fortpflanzung der Castanelliden. Zool. Jahrb., Abt. f. Anat. u. Ontog., Bd. XXVII, S. 243-280, 3 Taf , 5 Textabb.

Zuelzer, M., Bau und Entwicklung von Wagnerella borealis Mereschk. Arch. f. Protistenk., Bd. XVII, S. 135-202, 5 Taf., 20 Fig. im Text.

Coelenteraten:

Bigelow, H. B., Cruise of the U. S. Fisheries Schooner "Grambus" in the Gulfstream during July, 1908, with Description of a new Medusa (Bythotiaridae). Bull. Mus. Comp. Zool. Harvard College, Vol. LII, Nr. 12, S. 195-212, 1 Taf.

Bigelow, H. B., Reports on the Scienti fic Results of the Expedition to the Wastern Tropical Pacific, in charge of Alexander Agassiz, by the U. S. fish Commission Steamer Albatross. XVI. The Medusae. Memoirs Mus. Com. Zoolog. Harvard College, Vol. XXXVII, 243 S., 48 Taf.

Kühn, A., Sproßwachstum und Polypenknospung bei den Thecaphoren. Studien zur Ontogenese und Phylogenese der Hydroiden. Zool. Jahrb., Abt. f. Anat. u. Ontog., Bd. XXVIII, Heft 2, S. $387 \div 476,6$ Taf., 22 Fig. im 'Text.

Lipin, A., Ober den Bau des Süßwasser-Cölenteraten Polypodium hydriforme Uss. Zool. Anzeiger, Bd. XXXIV, S. 346-346, 9 Textfig.

Pax, Ferd., Aktinienstudien 1. Polyparium ambulans. Jenaer Zeitschr. f. Naturwiss., Bd. XLV, S. $325-344,1$ Taf., 3 Fig. im Text.

Pax, Ferd., Die Aktinien der ostafrikanischen Inseln. Voeltzkows Reise in Ostafrika in den Jahren 1903-1905, Bd. II, 20 S., 3 Taf.

Ritter, Wm. E., Halocynthia Johnstoni n. sp. A comprehensive inquiry as to the extent of law and order that prevails, in a single animal species. University of California Publications in Zoology, Vol. VI, S. 65-114, 8 Taf.

Roule, L., Liste des Antipathaires et des Cérianthaires provenant des récentes compagnes de It Princesse Alice. Bull. Inst. Océan. Monaco, Nr. 134, $5 \mathrm{~S}$.

Topsent, E., Etude sur quelques Cladorhiza et sur Euchelipluma pristina n. gen. n. sp. Bull. Inst. Océan. Monaco, Nr. 15̃1, 21 S., 2 Taf,

Torrey, H. B., and Kleeberger, F.L., Three species of Cerianthus from Southern California. University of California Publications in Zoology, Vol. VI, S. 115-125, 4 Fig. im Teyt.

Torrey, H. B., The Leptomedusae of the San Diego Region. Ibidem, S, 11-31, 11 Textfiguren.

v. Uexkiill, J., Résultats des recherches effectuées sur les tentacules de l'Anemonia sulcata, au Musée Océanographique de Monaco, en décembre 1908 (note préliminaire). Bull. Inst. Océan. Monaco, Nr. $148,3 \mathrm{~S}$.

Will, L., Die Klebkapseln der Aktinien und der Mechanismus ihrer Entladung. Sitz.Ber. u, Abh. d. naturf. Gesellschaft zu Rostock, N. F., Bd. I. 30 S., 2 Tai.

Will, L., Uber das Vorkommen kontraktiler Elemente in den Nesselzellen der Cölenteraten. Sitzungsber, d. Naturf. Gesellsch, zu Rostock, 20 S., 2 Taf.

\section{Echinodermen:}

Baltzer, F., Uber die Entwicklung der Echinidenbastarde mit besonderer Berücksichtigung der Chromatinverhältnisse. Zool. Anz., Bd. XXXV, S. 7--15, 2 Fig., 2 Tab.

Herbst, C., Vererbungsstudien VI. Die zytologischen Grundlagen der Verschiebung der Vererbungsrichtung nach der mütterlichen Seite, 1. Mitteilung. Arch. f. Entwickl.Mech., Bd. XXVII, Heft 2, 32 S., 4 Taf.

Hérouard, E., Triconus nouveau genre de la famille des Psychropotineae. [TiefseeHolothurien.] Bull. Inst. Océan. Monaco, Nr. 145, 5 S.

Kapelwieser, H., Entwicklungserregung bei Seeigeleiern durch Molluskensperma. Arch. f. Entwickl.-Mech., Bd. VII, S. $434-462,3$ Taf., 3 Fig. im Text.

Me Clendon, J. F., The Ophiurans of the San Diego region. Univers. Cal. Publ. Zoology, Bd VI. S. $33-64,6$ Taf.

Fermes:

De Beanchamp, P., Recherches sur les Rotifères. Arch. de Zoologie expériment. et générale (1908) 1909, T. V, Nr. 1, 410 S., 9 Taf. [Sehr wertvolle und umfassende Monographie, behandelt Okologie, Körperbedeckung und Räderorgane; Autoreferat in Band III dieser Revue.]

Dawydofi, C., Sur la Régéneration de l'extrémité posterieure chez les Némertiens. Bull. Acad. Imp. Sc. St. Pétersbourg, 11 S., 12 Fig. im Text. 
Faurel, $\mathbf{P}$, Deuxième note préliminaire sur les Polychètes provenant des compagnes de l'Hirondelle et de la Princesse-Alice on déposées dans le Musée Océanographique de Monaco. Bull. Inst. Océan. Monaco, Nr. 142, $76 \mathrm{s.}$

Goldschmidt, Rich., Eischale, Schalendrüse und Dotterzellen der Trematoden. Zoolog. Anz., Bd. XXXIV, S. 481-498, 10 Fig. im Text.

Gravey, F. H., Studies on Polychaet Larvae. Quart. Journ. Micr. Sc., Vol. LIII, part 3 , 31 S., 1 Taf.

Meisenheimer, Joh., Die Exkretionsorgane der wirbellosen Tiere. I. Protonephridien $u$. typische Segmentalorgane. Frgebnisse u. Fortschritte d. Zoolog., Bd. II, Heft 2, S. $275-366,37$ Fig.

Moore, J. Percy, The Polychaetous Annelids dredged by the U. S. S. "Albatross" of the coast of Southern California in 1904, I. Syllidae, Sphaerodoridae, Hesionidae and Phyllodocidae. Proc. Acad. Nat. Sc. Philadephia, 31 S., 2 Taf.

Moore, J. Perey, The Polychaetous Annelids dredged in 1908 hy Mr. Owen Bryant off the coasts of Taborador, Newfoundland, and Nova Scotica. Proc. U. S. Nat. Mus., Vol. XXXVII, $14 \mathrm{~s}$.

Moore, J. Percy, Polychaetons Annelids from Monterey Bay and San Diego, California. Proc. Acad. Nat. Se. Philadelphia, 31 S., 3 Taf.

Rauther, Morphologie und Verwandtschaftsbeziehungen der Nematoden und einiger ihnen nahe gestellter Vermalien. Ergebn. u. Fortschr, d, Zoolog., Bd. II.

salensky, W. Embryonale Hntwieklung des Prosorochmus viviparus (Monopora vivipara). Bull. de l'Acad. Imp. des Sc. St. Pétersbourg 1909, S. 325-340, 9 'T'extfig.

Steinmann, P., Zur Polypharyngie der Planarien. Zool. Anz., Bd. XXXV, S. 161-165, 2 Fig. im Text.

Steinmann, P., Untersuchungen an neuen Tricladen. Zeitschr. f, wiss. Zoolog., Bd. XCIII, S. $157-184,1$ Taf.

Voigt, M., Nachtrag zur Gastrotrichenfauna Plöns. Zool. Anz., Bd. XXXII, S. 717-722. 3 Fir.

Wilhelmi, J., kirnährungsweise, Gelegenheits-, Pseudo- und Dauerparasitismus bei Seetricladen. Zool. Anz., Bd. XXXIV, S. $723-730$.

Wilhelmi, J., Zur Regeneration und Polypharyngie der'Tricladen. Zool An\%., Bd. XXXIY, S. $673-677$.

Wilhelmi, J., Nachtrag zur Mitteilung über die Jolypharyngie der Tricladen. Zool. Anz., Bd. XXXV, S. $311-317,1$ Fig.

Mollusken und Prochordaten:

Baner, V., Hinführung in die Physiologie der Cephalopoden. Mit besonderer Berücksichtigung der im Mittelmeer häufigen Formen. Mitteil. d. Stat. Neapel 1909, Bd. XIX, 2, mit 31 Textfig. u. 3 Taf.

(rariaeff, Wl., Zur Histologie des zentralen Nervensystems der Cephalopoden. Zeitschr. f. wiss. Zool., Bd. XCII, S. 149-186, 2 Taf.

Guérin-Ganivet, J., et Joubin, L., Notes préliminaires sur les gisements de Mollusques comestibles des Cotes de France. Nr. 131: L'Estuire de la Gironde (avec une carte). Nr. 135: La côte des Landes de Gascogne et le Bassin d'Arcachon (avec deux cartes). Nr. 136: I a côte de Lannion à Tréguier (avec une carte). - Nr. 139: La côte de 'Tréguier à Paimpol; l'île de Bréhat. - Nr. 141: La baie de Saint-Brieuc. Bull. Inst. Océan. Monaco.

Heider, K., Zur Entwicklung von Balanoglossus clavigerus Delle Chiaje. Zool. Anzeigrr, Bd. XXXIV, S. $675-704,14 \mathrm{Abb}$.

Martini, E., Studien über die Konstanz histologischer Elemente. 1. Oikopleura longicauda. Zeitschr. f. wiss. Zool., Bd. XCII, S. 564-626, 3 Taf., 22 Textfig.

Milascewicz, K. O., Liste des Mollusque, collectionnés durant l'expédition zoologique de Mr. S. A. Zernov dans la partie $\mathrm{NW}$ de la mer Noire à bord du Cateau „Académicien Baer". Extrait de l'Annuaire du Musée Zool. de l'Academie Imp. des Sciences St. Pétersbourg, 'T'. XIV, S. 145-166, 1 Textfig.

Steusloff, U., Paludestrina jenkinski Smith an der deutschen Ostseeküste. Arch. d. Ver. d. Fr. d. Naturgeseh. Mecklenburgs, Bd. LCIII, s. $82-93$, 1 'Taf.

Crustaceen:

Breckner, A.. Vorläufige Mitteilungen über experimentelle Untersuchungen an Artemia salina. Verh. u. Mitt. d. Siebenbürg. Vereins f. Naturw., Bd. LVIII, 53 s., 1 Taf.

Brehm, Y., Copepoden aus den phlegränischen Feldern. Zool. Anz. 
Cherreux, E., Diagnoses d'Amphipodes nouveaux provenant les campagnes de la Princesse-Alice dans l'Atlantique nord. Bull. Inst. Océan Monaco, Nr. 150, $7 \mathrm{~s}$.

Cherrenx, E., Biospeologica: Amphipodes I. Arch. Zoologie Exper. et Générale, 5. Sér.. I. II, S. 27-.2,2 Taf.

Keilhack, L., Bemerkungen zur Systematik und Nomenklatur der Cladoceren und Malakostraken der deutsehen Binnengewässer. Zool. Anz., Bd. XXXIV, S. 324-329.

Keilhack, I.., Zur Nomenklatur der deutschen Phyllopoden. Zool. Ann.: Zeitsch, f. Gesch. Zool.. Bd. III, S. 177-184.

Keilhack, L., Bemerkenswerte Cladoceren und Copepoden aus den Dauphiné-Alpen. 3. Beitrag zur Kenntnis der SüBwasserfauna der Jauphiné-Alpen. Arch. f. Hydrobiologie $\mathrm{u}$. Planktonk., $2 \mathrm{~S}$.

Keilhack, L., Die Ephippien der Macrothriciden. 2. Beitrag zur Kenntnis der Süßwasserfuuna der Dauphiné-Alpën, 4 S., 1 Taf.

Langhans, V. H., Über experimentelle Untersuchungen zu Fragen der Fortpflanzung, Variation und Vererbung bei Daphniden. Verhandl. d. D. Zool. Ges. 1909, S. 281-291.

Minkiewiez, R., llémoire sur la biologie du Tonnelier de mer, Phronima sedentaria. Forsk, avek 21, et 16 Fig. Bull. Inst. Océan Maraco Nr. 146 u. $152,21+19 s$.

Neresheimer, E., Studien über Süsswasser-Lernaeopodiden. Ber. d. Bayer. Biolog. Versuchsst. in München, Bd. Ir, 9 S., 1 Taf., 1 Textfig.

Rühe, F. E., Bemerkungen über das Vorkommen der Bosmina obtusirostris in Norddeutschland. Zool. Anz., Bd. XXXIV, 3 S., 3 Fig.

Sars, G. O., Note préliminaire sur trois formes remarquables de Copépodes de S. A. S. le Prince Albrecht de Monaco, avec 3 figures. Bull. Inst. Océan Monaco, Nr, 147, 8 S.

sehleip, W., Vergleichende Untersuchung der Eireifung bei parthenogenetisch und bei geschlechtlich sich fortpflanzenden Ostrakoden. Archiv f. Zellforschung, Bd. II, S. $390-431,3$ Taf.

StranB, E., Das Gammaridenauge. Studien über ausgebildete und rückgebildete Gammaridenaugen. Wissensch. Ergebn. d. deutsch. Tiefsee-Exped., Bd. XX, 84 S., 6 'Taf., 47 Fig. im Text.

Tattersall, W. M., The Schizopoda collected by the Maia and Puritan in the Meditteranian. Mitt. d. Zool. Stat. Neapel, Bd. XIX, Heft 2, S. 117-143, I Taf,

Taube, E., Beiträge zur Entwicklungsgeschichte der Euphausiden. 1. Die Befruchtung des Eies bis zur Gastrulation. Zeitschr. f. wissenschaftl. Zool., Bd. XCII, S. 427-464, 2 Taf., 6 'T'extfig.

Tollinger, J., Der Verdanungstrakt von Lynceus intermedius (G. O. Sars.) Ann. de Biologie lacustre, tome III, $30 \mathrm{~s}$., 2 Taf.

v. Uexkiill, et Gross, Résultats des recherches effectuées sur les extrémités des langroustes et des crabes, au Musée Océanographique de Monaco, en février et en mars 1909, (note préliminaire). Bull. Inst. Océan. Monaco, Nr. 149, $4 \mathrm{~S}$.

Woltereck, R., Reports on the Scient. Results of the Expedition to the Eastern Trop. Pacific, in charge of Al. Agassiz, by the U. S. fish Commission Steamer "Albatross", XVIII, Amphipoda (I: Hyperidea Gammaroidea Primitiva). Bull. Mus. Comp. Zoolog. Harvard College, Vol. LXX, $24+8$ S., 8 Taf.

Woltereck, R., Weitere experimentelle Untersuchungen über Artveränderung, speziell über das Wesen quantitativer Artunterschiede bei Daphniden. Verhandl. d. D.Zool. Ges. 1909, S. 110-172, 18 Textfig. [Referat in dieser Revue, S. 770.]

Zernov, (Sernof) (Sernov) S. A., Penilia schmackeri Richard (Cladocere) dans la Mer Noire. Note préliminaire. Extrait de l'Annuaire du Musée Zool. de l'Acad. des Sciences St. Pétersbourg, tome XIII, 1908, 3 S.

Insekten und Milben:

Boerner, C., Jie Tracheenkiemen der Ephemeriden. Zool. An\%., Bd. XXXIJI. S. 80f--823, 4 Fig.

Brocher, F., Recherches sur la respiration des insectes aquatiques adultes. Ann. Biol. lacustre tome IV, S, 9-32, 7 Taf.

Brocher, F., Sur l'organe pulsatile observé dans les pattes des hémiptères aquatiques. Ibidem, $\$ .33-41,2$ Fig.

Brocher, F., Métamorphoses de Tipula lunata Lin. Ibidem, S. 42-43, 2 Fig.

Brucher, F., Métamorphoses de l'Hemerodromia praecatoria Fall. Ibidem, S. 44-45, 2 Fig.

Rousseau, E., Etude monographique des larves des Odonates d'Europe. Ann. Biol. lacustre, tome III, S. $300-366,47$ Fig. im Text. 
Thienemann, A., Beitrăge zur Kenntnis der westfälischen SüBwasserfauna I. Chironomiden von. J. J. Kieffer u. A. Thienemann. Bd. XXXVII, Jahresber. d. westfäl. Prov.Ver. für Wissensch. u. Kunst, $8 \mathrm{~S}$.

Thienemann, A., Prestwichia aquatica Lubbock. Zeitschr. wiss. Insektenbiol,, IBd. V, Heft $10,1 \mathrm{~S}$.

Walter, C., Neue Liste belgischer Hydracarinen. Ann. Biol. lacustre, tome IV, S. 46-52.

Zülzer, M., Beitrag zur Kenntnis der Entwicklung von Psychoda sexpunctata, der Schmetterlingsmücke. Mitt. a. d. Kgl. Prüfungsanstalt f. Wasserversorgung etc. Berlin, H. 12, S. 213-224, 2 Taf.

Vertebraten, vorzugsweise Fische und Fischerei:

Brühl, L., Altersbestimmmung bei Fischen vor 150 Jahren. Fischerei-Zeitung Bd. XIII, S. $39-40$.

Brübl, L., Die Fischerei-Verhältnisse an der Westküste von Grönland. Deutsche FischereiZeitung, 32. Jahrg, 29 S., 1 Karte.

Dafert, F. W. und Kornauth, K., Bericht über die Tätigkeit der k. k. land.-chemischen Versuchsstation und der mit ihr vereinigten $\mathbf{k}$. k landw.-bakteriolog. Pflanzenschutzstation in Wien. Zeitschr. f. landwirtsch. Versuchswesen in Osterreich, $100 \mathrm{~S}$. [Enthält den Bericht der Fischereiabteilung.]

Damas, D., Contribution à la Biologie des Gadides. Rapports et Procies-Verbaux du Conseil Intern. pour l'exploration de la Mer, vol. X, 277 S., 21 Taf., 25 Fig.

Ehrenbaum, E., Eier und Larven der im Winter laichenden Fische der Nordsee. II. Die Laichverhältnisse von Scholle und Flunder. Arb. d. Deutsch. wiss, Kommission f. d. intern. Meeresforschung. B. Aus d. biol. Anst. Helgoland, Nr. 12, S. 145-176, 7 Karten, 1 Fig. im Text u. 6 Tabellen, ferner Bd. XX, Fangtabellen von E. Ehrenbaum u. W. Mielck.

Ehrenbaum, E., Eier und Larven von Pleuronektiden der Nordsee und benachbarter Gewässer. Neuere Untersuchungen aus den Jahren 1904-1909. Rapports et ProcèsVerbaux du Conseil intern. pour l'exploration de la mer, Vol. XII, 32 s.

Gast, R., Die Entwicklung des Okulomotorius und seiner Ganglien bei Selachier-Embryonen. Mitt. aus d. Zool. Stat. Neapel, Bd. XIX, Heft 3, S.270--444, Taf. 12--16.

Giinther L., Fischkost. Constrōms Verlag, Hamburg. $6 \mathrm{~S}$.

Guérin Ganivet, Note sirr la présence du Tropidonotus natrix dans l'eau de mer. Bull. Inst. Océan. Monaco, Nr. 132, $2 \mathrm{~S}$.

Hein, W., Gitterkiesbett u Irehstromapparat. Allgem. Fischereizeitg., Bd. XXXIV, 6 S.

Helland-Hansen, B., Statistical Research into the biology of the haddock and cod in the north sea. Rapports Conseil intern. Explor. Mer. Vol. X, 62 S., 34 Fig.

Kükenthal, W., Untersuchungen an Walen. Jen. Zeitschr. f. Naturw. Bd. XLV, S. 540-588,8 Taf., 6 Fig. im Text.

Levander, K. M., Beobachtungen über die Nahrung und die Parasiten der Fische des Fimnischen Meerbusens. Finnl. hydrogr. Untersuch., Nr. 5, $44 \mathrm{~S}$.

Lübbert, W., Die deutsche Hochseesegelfischerei in Vergangenheit und Gegenwart. Meereskunde, Jahrg. III, 35 S., $23 \mathrm{Abb}$.

Luther, Alex., Beiträge zur Kenntnis̃ von Muskulatur und Skelett des Kopfes des Haies Stegostoma tigrinum Gm. und der Holocephalen mit einem Anhang über die Nasenrinne. Acta soc. sc. Fennicae, 'T' XXXVII, Nr. 6, $60 \mathrm{~s}$.

Lnther, Alex., Untersuchungen über die vom Nervus trigeminus innervierte Muskulatur der Selachier (Haie und Rochen) unter Berücksichtigung ihrer Beziehung zu benachbarten Organen. Acta Soc. Scientiarum Fenricae, T. XXXVI, 176 S., 5 Doppeltaf., 23 Fig. im Text.

Yeresheimer, E., Blutsverwandtschaft und Serumreaktion bei Salmoniden. Ber. d. Kgl. Bayer. Biol. Versuchsstat. München, Bd. II, S. 79-88.

ReuB, H., Die Wirliung der Kohlensäure anf Fische. Ibidem, S. 89-142, 10 Abb.

Schneider, G., Über das Wachstum der Aale (Angnilla vulgaris Flem.) in den Gewässern Schwedens. Publications de Circonstance, Nr. 46, 18 S., 1 Textfig.

steche, 0., Die Leuchtorgane von Anomalops katoptron und Photoblepharon palpebratus,

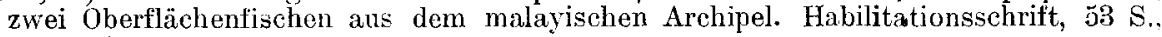
7 Fig. im Text.

Vinciguerra, Decio Congresso internazionale di pesea tenuto in Washington nel 1908 con notizie sulla pesca e la piscicoltura negli Stati Uniti, Rom, 30 S., 7 Figuren im Text. 